\title{
A novel validated model for the prediction of insulin therapy initiation and adverse perinatal outcomes in women with gestational diabetes mellitus
}

\author{
Robyn A. Barnes ${ }^{1}$. Tang Wong ${ }^{1,2}$ - Glynis P. Ross ${ }^{1,3}$ • Bin B. Jalaludin ${ }^{4,5}$. \\ Vincent W. Wong ${ }^{2,6}$ - Carmel E. Smart ${ }^{7,9}$ - Clare E. Collins ${ }^{8,9}$ • \\ Lesley MacDonald-Wicks ${ }^{9}$ • Jeff R. Flack ${ }^{1,2,10}$
}

Received: 31 March 2016 / Accepted: 15 June 2016 /Published online: 8 July 2016

(C) Springer-Verlag Berlin Heidelberg 2016

\begin{abstract}
Aims/hypothesis Identifying women with gestational diabetes mellitus who are more likely to require insulin therapy vs medical nutrition therapy (MNT) alone would allow risk stratification and early triage to be incorporated into risk-based models of care. The aim of this study was to develop and validate a model to predict therapy type (MNT or MNT plus insulin [MNT+I]) for women with gestational diabetes mellitus (GDM).

Methods Analysis was performed of de-identified prospectively collected data (1992-2015) from women diagnosed with GDM by criteria in place since 1991 and formally adopted and promulgated as part of the more detailed 1998 Australasian Diabetes in Pregnancy Society management guidelines. Clinically relevant variables predictive of insulin therapy by univariate analysis were dichotomised and included in a multivariable regression model. The model was tested in a separate clinic population.

Results In 3317 women, seven dichotomised significant independent predictors of insulin therapy were maternal age $>30$ years, family history of diabetes, pre-pregnancy obesity $\left(\mathrm{BMI} \geq 30 \mathrm{~kg} / \mathrm{m}^{2}\right.$ ), prior GDM, early diagnosis of GDM ( $<24$ weeks gestation), fasting venous blood glucose level

Robyn A. Barnes

Robyn.Barnes@sswahs.nsw.gov.au

1 Diabetes Centre, Bankstown-Lidcombe Hospital, 68 Eldridge Road, Bankstown, NSW 2200, Australia

2 Faculty of Medicine, University of New South Wales, Sydney, NSW, Australia

3 Faculty of Medicine, University of Sydney, Sydney, NSW, Australia

4 Epidemiology Department, Healthy People and Places Unit, South Western Sydney Local Health District, Liverpool, Australia

5 Ingham Institute for Medical Research, University of New South Wales, Liverpool, NSW, Australia
\end{abstract}

$(\geq 5.3 \mathrm{mmol} / \mathrm{l})$ and $\mathrm{HbA}_{1 \mathrm{c}}$ at GDM diagnosis $\geq 5.5 \%$ ( $\geq 37 \mathrm{mmol} / \mathrm{mol}$ ). The requirement for $\mathrm{MNT}+\mathrm{I}$ could be estimated according to the number of predictors present: $85.7-93.1 \%$ of women with 6-7 predictors required MNT+I compared with $9.3-14.7 \%$ of women with $0-1$ predictors. This model predicted the likelihood of several adverse outcomes, including Caesarean delivery, early delivery, large for gestational age and an abnormal postpartum OGTT. The model was validated in a separate clinic population.

Conclusions/interpretation This validated model has been shown to predict therapy type and the likelihood of several adverse perinatal outcomes in women with GDM.

Keywords Clinical diabetes $\cdot$ Health-care delivery $\cdot$ Insulin therapy $\cdot$ Models $\cdot$ Nutrition and diet $\cdot$ Pregnancy

\section{Abbreviations \\ ADIPS Australasian Diabetes In Pregnancy Society \\ BGL Blood glucose level}

6 Liverpool Diabetes Collaborative Research Unit, Ingham Institute of Applied Science, Liverpool, NSW, Australia

7 Department of Paediatric Endocrinology and Diabetes, John Hunter Children's Hospital, Newcastle, NSW, Australia

8 Priority Research Centre in Physical Activity and Nutrition, Faculty of Health and Medicine, The University of Newcastle, Callaghan, NSW, Australia

9 School of Health Sciences, Faculty of Health and Medicine, The University of Newcastle, Callaghan, NSW, Australia

10 School of Medicine, Western Sydney University, Sydney, NSW, Australia 


$\begin{array}{ll}\text { DCDS } & \text { Diabetes Centre Database System } \\ \text { GDM } & \text { Gestational diabetes mellitus } \\ \text { LGA } & \text { Large for gestational age } \\ \text { MNT } & \text { Medical nutrition therapy } \\ \text { MNT+I } & \text { Medical nutrition therapy plus insulin } \\ \text { NPV } & \text { Negative predictive value } \\ \text { PPV } & \text { Positive predictive value } \\ \text { ROC } & \text { Receiver operating characteristic } \\ \text { SGA } & \text { Small for gestational age } \\ \text { SMBG } & \text { Self-monitored blood glucose }\end{array}$

\section{Introduction}

Insulin resistance and a reduced pancreatic beta cell reserve contribute to the development of gestational diabetes mellitus (GDM) [1]. GDM varies in severity, with some women requiring insulin therapy in addition to mainstay medical nutrition therapy (MNT) [2].

Rapidly increasing GDM rates present multidisciplinary teams with the challenge of providing optimal diabetes education and management services when clinical resources are constrained. Different models of care are developing internationally in an attempt to meet the increasing demand for GDM services. Identifying women with GDM who are more likely to achieve optimal clinical outcomes by MNT alone vs those who may require MNT plus insulin $(\mathrm{MNT}+\mathrm{I})$ could enable early triage to be incorporated into risk-based models of care.

A number of studies have successfully identified one or more clinical or biochemical characteristics present at the time of GDM diagnosis that are associated with the need for insulin treatment [3-12]. These include early GDM diagnosis (e.g. at $<25$ weeks gestation), a family history of diabetes, nonEuropean ethnicity, an older age, elevated fasting blood glucose level (BGL) and $\mathrm{HbA}_{1 \mathrm{c}}$ at GDM diagnosis, and an elevated pre-pregnancy BMI [3-12]. Despite the identification of individual independent predictors of the need for insulin therapy in GDM, only a few studies have attempted to use these factors to develop a model for predicting therapy type (either MNT or MNT+I) in women with GDM [3, 5, 11]. For those that have made such an attempt, success has been limited.

The aim of the current study was to develop and validate a clinically useful model to predict the need for insulin therapy in addition to MNT in the management of women with GDM. The working hypothesis was that commonly available clinical and biochemical variables could be incorporated into a model that could predict therapy type in women with GDM.

\section{Methods}

The study population comprised women with GDM attending Bankstown-Lidcombe Hospital Diabetes Centre, New South
Wales, Australia, between 1992 and 2015. This 458 bed metropolitan teaching hospital is situated in an ethnically diverse region with high numbers of residents with East and SouthEast Asian, Middle Eastern, and South Asian backgrounds. Socioeconomic disadvantage is common in this area and the degree of health literacy is highly variable. Compared with the state of New South Wales, Bankstown Local Government Area has a lower percentage of Australian-born individuals $(56.2 \%$ vs $68.6 \%)$, more unemployment $(7.6 \%$ vs $5.9 \%)$ and a lower median weekly household income (2011 National Census Data) [13].

De-identified data that were prospectively collected as part of routine heath care were extracted from a customised database (Bankstown-Lidcombe Hospital Diabetes Centre Database System [DCDS]). The data were analysed for singleton births in women diagnosed with GDM by criteria in use from 1991 [14] and formally adopted and promulgated as part of the more detailed 1998 Australasian Diabetes In Pregnancy Society (ADIPS) management guidelines [15], with any BGL result of fasting $\geq 5.5 \mathrm{mmol} / 1$ and/or $2 \mathrm{~h} \geq 8.0 \mathrm{mmol} / \mathrm{l}$ in a $75 \mathrm{~g}$ OGTT (ADIPS Australian criteria). Universal screening was offered at 24-28 weeks gestation via a $50 \mathrm{~g}$ oral glucose challenge, proceeding to a $75 \mathrm{~g}$ OGTT if the $1 \mathrm{~h}$ BGL was $\geq 7.8 \mathrm{mmol} / \mathrm{l}$. Women at a high risk of GDM underwent earlier screening that was repeated at 24-28 weeks if the early test was normal. From 2010 onward, a $1 \mathrm{~h}$ value of $\geq 10.0 \mathrm{mmol} / 1$ in the OGTT was also considered diagnostic for GDM.

MNT was the first-line therapy, with insulin commenced if optimal glycaemic targets (defined as fasting BGL $<5.5 \mathrm{mmol} / \mathrm{l}, 1 \mathrm{~h}$ postprandial BGL $<7.4 \mathrm{mmol} / 1$ and $2 \mathrm{~h}$ postprandial $\mathrm{BGL}<7.0 \mathrm{mmol} / \mathrm{l}$ ) were not reached. For women newly diagnosed with GDM, if BGL glycaemic targets were not reached within 2 weeks, then insulin therapy was commenced. However, insulin was commenced at any time during pregnancy when a sustained achievement of glycaemic targets by MNT alone failed to occur. Oral hypoglycaemic agents were not used. Individuals without all data items required for inclusion in the model or a multiple pregnancy were excluded.

Clinical management of GDM English-speaking women received initial education from a dietitian and diabetes educator, in either an individual (1992 to 31 January 2012) or group (1 February 2012 to 1 March 2015) setting. All non-Englishspeaking women received initial education in an individual setting via an interpreter. All initial education was followed by at least one individual dietitian appointment, usually within 6-8 days. The women self-monitored their BGLs (i.e. selfmonitored blood glucose [SMBG]) four times daily: after fasting and either 1 or $2 \mathrm{~h}$ after the start of three main meals, based on the postprandial value that was most elevated in the OGTT. One hour postprandial testing was used after the introduction of a $1 \mathrm{~h}$ diagnostic value for the $75 \mathrm{~g}$ OGTT from 
2010 onward. Each woman was monitored in the multidisciplinary clinic for the remainder of her pregnancy. SMBG results were reviewed by the diabetes educator and endocrinologist at each visit, and insulin was commenced and/or titrated if glycaemic targets were not reached at any time before childbirth. Women were weighed at each visit.

MNT All women were advised to follow a carbohydrate controlled diet with higher fibre (25-30 g/day) and lower saturated fat (7-10\% of total energy). Dietary advice aimed for approximately $45-50 \%$ of energy to be obtained from carbohydrate, $20-25 \%$ from protein and $25-30 \%$ from fat. Women were advised to spread their carbohydrate intake over three main meals and three snacks, and to consume approximately $30-45 \mathrm{~g}$ carbohydrate at breakfast, $45-60 \mathrm{~g}$ at lunch and dinner, and $15-30 \mathrm{~g}$ in snacks. Women were also advised to minimise their intake of concentrated sources of fat and added sugars in their diet. Lower glycaemic index foods were recommended. Regular physical activity of approximately $30 \mathrm{~min} /$ day was promoted. All women received individualised MNT education within the multidisciplinary GDM clinic after their initial education. Individualised MNT advice was based on individual dietary requirements, personal food preferences, reported dietary intake (assessed by diet history or 3 day food record), and SMBG results. Maternal weight gain recommendations were stratified according to pre-pregnancy BMI, in accordance with the Institute of Medicine guidelines [16, 17].

Clinical variables The following clinical variables were analysed or derived from items in the database (DCDS): age, gravida, parity, ethnicity, pre-pregnancy BMI (based on selfreported pre-pregnancy weight and measured height), history of GDM, family history of diabetes, OGTT results and $\mathrm{HbA}_{1 \mathrm{c}}$ at GDM diagnosis. The treatment modality (MNT or MNT+I) during pregnancy, mode of delivery, birth-weight and presence of delivery complications were recorded in the database as part of routine care.

To determine whether the therapy prediction model also predicted the likelihood of adverse perinatal outcomes, these outcomes were compared with the number of therapy predictors for each pregnancy. Adverse perinatal outcome assessments included rates of early delivery ( $<37$ weeks), Caesarean section, neonatal hypoglycaemia, large for gestational age (LGA) infants and small for gestational age (SGA) infants. Birth-weights were categorised into SGA $(<10$ th percentile) and LGA ( $>90$ th percentile) by reference to customised percentile charts which adjust for age, maternal height (measured by stadiometer), self-reported pre-pregnancy maternal weight (recorded at first GDM clinic visit), parity and ethnicity using the www.gestation.net website and the method of Gardosi et al (Bulk Centile Calculator V6.7.3 -13) [18]. Postpartum OGTT results were included for women who had completed the test (55.0\% of the total).
Ethical approval for this study was provided by the South Western Sydney Local Health District Research and Ethics Committee.

Statistical analysis Readily available clinical and biochemical variables were initially assessed as predictors of insulin therapy in single variable logistic regression models, either as yes/no categorical variables or continuous variables. Variables with a $p$ value of $<0.20$ in single variable logistic regression models were included in the initial multivariable logistic regression model. The backward selection method was then used to derive the final model consisting of seven significant predictors $(p<0.05)$. None of the cut-off points used in the model were pre-specified: all emerged from the data. Once significant variables were identified by multivariable regression analysis, continuous variables were dichotomised for ease of use in a clinical setting. Each continuous variable was first categorised into four or five groups and cross-tabulated against therapy type. The point with the greatest increase in insulin therapy type was then identified and tested as a significant predictor of insulin use by univariate analysis $(p<0.05)$. The variation in the logistic regression models explained by each potential predictor was assessed using the Nagelkerke $R^{2}$ value.

Seven significant predictors for treatment were included in the final logistic regression model. A 'predictor score' $(0-7)$ was calculated for each participant based on the seven significant predictors of therapy outcome. For example, if a participant had three of the seven predictors, then they received a 'predictor score' of three. To further evaluate the final predictor model, a receiver operating characteristic (ROC) curve of sensitivity plotted against 1 -specificity was constructed based on the number of predictors present $(0-7)$ vs the therapeutic outcome (MNT vs MNT+I).

Continuous data were compared between study groups using independent two-tailed $t$ tests. Categorical data were analysed using $2 \times 2$ contingency tables and $\chi^{2}$ tests. Results are presented as the mean \pm SD or ORs and associated $95 \%$ CIs. The sensitivity, specificity, positive predictive value (PPV) and negative predictive value (NPV) of the model were also calculated.

All analyses were done in IBM SPSS Statistics software (version 22.0, Chicago, IL, USA). Statistical significance was set at a $p$ value of $<0.05$.

The final model was subsequently validated in a second clinic population of women $(n=1040)$ with GDM who were managed from 2009 to 2013 at the Liverpool Hospital Diabetes Centre, NSW, Australia. This population differed in ethnic composition, percentage MNT+I and model of care. Initial education was delivered in a group setting: one to one MNT education was not routinely offered. In addition, those managed with MNT alone had follow-up provided by diabetes educators, whereas those on insulin were managed by an endocrinologist. 


\section{Results}

Of 4015 women, a total of 3317 had complete data on all items assessed for inclusion in the model. Those with complete data were diagnosed at a mean \pm SD gestation of $26.7 \pm 5.9$ weeks and had a mean age of $32.1 \pm 5.4$ years, an OGTT fasting BGL of $5.1 \pm 0.8 \mathrm{mmol} / \mathrm{l}, \mathrm{HbA}_{1 \mathrm{c}}$ of $5.3 \pm 0.60 \%(34 \pm 6.6 \mathrm{mmol} / \mathrm{mol})$ and a pre-pregnancy BMI of $26.4 \pm 6.3 \mathrm{~kg} / \mathrm{m}^{2}$. Their ethnic background was $33.7 \%$ East and South-East Asian, 27.0\% Middle Eastern, 22.5\% European, 11.5\% South Asian and 5.3\% African subcontinent or Pacific Islander.

Table 1 summarises the demographic and clinical data from the 4015 women whose clinical and laboratory data were used to develop the model. Gravida, parity, prior GDM, previous fetal macrosomia, a family history of diabetes, ethnicity, maternal age, nulliparity, pre-pregnancy obesity (BMI $\geq 30 \mathrm{~kg} / \mathrm{m}^{2}$ ), gestational age at diagnosis, fasting and $2 \mathrm{~h}$ BGLs in the OGTT, and $\mathrm{HbA}_{1 \mathrm{c}}$ at GDM diagnosis were all found to be significant predictors of therapy type on univariate analysis, explaining $24.3 \%$ of the variance. A number of these lost significance when assessed in a multivariable regression model (gravida, parity, $2 \mathrm{~h}$ OGTT BGL, history of macrosomia and nulliparity) and were removed. In multivariable regression, the remaining eight variables explained $24.7 \%$ of the variance. Of these eight remaining variables, it was considered impractical to use ethnicity in a model for clinical use, and it was also removed. The use of ethnicity as a variable was considered impractical because of the large number of different ethnicities represented in these data; hence, including this variable would have prevented development of a model with a simple risk scoring system. The remaining seven variables still explained $24.2 \%$ of the variance, with five being continuous variables. The five continuous variables were then dichotomised: age $(\leq 30$ or $>30$ years); obesity (pre-pregnancy BMI $<30$ or $\geq 30 \mathrm{~kg} / \mathrm{m}^{2}$ ); gestational age at diagnosis $(<24$ or $\geq 24$ weeks gestation); fasting BGL $(<5.3$ or $\geq 5.3 \mathrm{mmol} / \mathrm{l})$, and $\mathrm{HbA}_{1 \mathrm{c}}$ at GDM diagnosis $(<5.5 \%[<37 \mathrm{mmol} / \mathrm{mol})$ or $\geq 5.5 \%[\geq 37 \mathrm{mmol} / \mathrm{mol}])$. These were added to the significant categorical variables family history of type 2 diabetes and previous GDM. In multivariable regression analysis, the seven dichotomous variables remained significant predictors in the final model, explaining $20.3 \%$ of the variance.

Table 2 shows the frequency with which the different numbers of predictors occurred and the corresponding percentage of women requiring MNT only vs MNT+I. Regarding the Bankstown-Lidcombe Hospital data, the greater the number of predictors present, the greater was the likelihood of MNT+I being prescribed. For those with $0-1$ predictors, the OR for remaining on MNT vs commencing insulin was $4.0(95 \%$ CI $3.2,4.9$ ), with a sensitivity of $30.9 \%$, a specificity of $89.9 \%$, a
Table 1 Clinical and laboratory variables associated with therapy type

\begin{tabular}{|c|c|c|c|}
\hline Variable & MNT only & $\mathrm{MNT}+\mathrm{I}$ & $p$ value \\
\hline Patients $(n[\%])$ & $2713(67.6)$ & $1302(32.4)$ & \\
\hline \multicolumn{4}{|l|}{ Clinical } \\
\hline Prior GDM & $532(19.6)$ & $431(33.1)$ & $<0.001$ \\
\hline Previous fetal macrosomia ( $>4 \mathrm{~kg}$ ) & $219(8.1)$ & $148(11.4)$ & $<0.001$ \\
\hline Family history of diabetes & $1462(53.8)$ & $873(67.0)$ & $<0.001$ \\
\hline Gravida & $2.8 \pm 1.9$ & $3.3 \pm 2.0$ & $<0.001$ \\
\hline Parity & $1.3 \pm 1.4$ & $1.6 \pm 1.6$ & $<0.001$ \\
\hline Ethnicity & & & $<0.001$ \\
\hline European & $559(20.6)$ & $336(25.8)$ & \\
\hline Middle Eastern & $706(26.0)$ & $431(33.1)$ & \\
\hline East/South-East Asian & $1020(37.6)$ & $298(22.9)$ & \\
\hline South Asian & $294(10.8)$ & $160(12.3)$ & \\
\hline Other & $137(5.0)$ & $78(6.0)$ & \\
\hline Age (years) & $31.8 \pm 5.5$ & $32.5 \pm 5.3$ & $<0.001$ \\
\hline Nulliparity & $945(34.8)$ & $380(29.2)$ & $<0.001$ \\
\hline Obesity (pre-pregnancy BMI $\geq 30 \mathrm{~kg} / \mathrm{m}^{2}$ ) & $468(17.7)$ & $487(38.1)$ & $<0.001$ \\
\hline Gestational age at diagnosis (weeks) & $27.9 \pm 5.3$ & $24.6 \pm 6.5$ & $<0.001$ \\
\hline \multicolumn{4}{|l|}{ Laboratory } \\
\hline Fasting BGL in OGTT $(\mathrm{mmol} / \mathrm{l})$ & $5.0 \pm 0.7$ & $5.5 \pm 1.0$ & $<0.001$ \\
\hline $2 \mathrm{~h} \mathrm{BGL}$ in OGTT $(\mathrm{mmol} / \mathrm{l})$ & $8.7 \pm 1.3$ & $8.9 \pm 1.7$ & $<0.001$ \\
\hline $\mathrm{HbA}_{1 \mathrm{c}}$ at GDM diagnosis (\%) & $5.2 \pm 0.6$ & $5.5 \pm 0.7$ & $<0.001$ \\
\hline $\mathrm{HbA}_{1 \mathrm{c}}$ at $\mathrm{GDM}$ diagnosis $(\mathrm{mmol} / \mathrm{mol})$ & $33 \pm 6.6$ & $37 \pm 7.7$ & $<0.001$ \\
\hline
\end{tabular}

Data are means $\pm \mathrm{SD}$ or $n(\%)$ 
Table 2 Number of predictors present and corresponding therapy type at both study sites

\begin{tabular}{llllll}
\hline $\begin{array}{l}\text { Number of } \\
\text { predictors present }\end{array}$ & \multicolumn{2}{l}{ Bankstown-Lidcombe Hospital Diabetes Centre } & & \multicolumn{2}{l}{$\begin{array}{l}\text { Liverpool Hospital } \\
\text { Diabetes Centre }\end{array}$} \\
\cline { 2 - 3 } \cline { 5 - 6 } & MNT only & & & MNT only & MNT+I \\
\hline 0 & $175(90.7)$ & $18(9.3)$ & & $55(87.3)$ & $8(12.7)$ \\
1 & $522(85.3)$ & $90(14.7)$ & & $109(69.9)$ & $47(30.1)$ \\
2 & $620(75.3)$ & $203(24.7)$ & & $136(63.8)$ & $77(36.2)$ \\
3 & $549(69.1)$ & $246(30.9)$ & & $79(56.5)$ & $100(43.5)$ \\
4 & $265(52.0)$ & $245(48.0)$ & & $28(23.5)$ & $91(76.5)$ \\
5 & $107(39.5)$ & $164(60.5)$ & & $12(20.7)$ & $46(79.3)$ \\
6 & $12(14.3)$ & $72(85.7)$ & $0(0.0)$ & $14(100.0)$ \\
7 & $2(6.9)$ & $27(93.1)$ & $549(52.8)$ & $491(47.2)$ \\
Total & $2252(67.9)$ & $1065(32.1)$ & &
\end{tabular}

Data are $n(\%)$
PPV of $86.6 \%$ and an NPV $38.1 \%$ for predicting that women with $0-1$ predictors would remain on MNT only (compared with those with 2-7 predictors). For those with 6-7 predictors, the OR for requiring $\mathrm{MNT}+\mathrm{I}$ vs remaining on MNT only was 16.4 (95\% CI 9.3, 28.8), with a sensitivity of $9.3 \%$, a specificity of $99.4 \%$, a PPV of $87.6 \%$ and an NPV of $69.9 \%$ for predicting a requirement for $\mathrm{MNT}+\mathrm{I}$ compared with those with $0-5$ predictors. The AUC of the ROC curve was 0.712 (95\% CI 0.693, 0.731).

To assess the model over time, we evaluated our data across two time periods (1992-2004 [ $n=1162]$ and 2005-2015 $[n=2155])$ and found similar prediction, with AUC values of $0.710(95 \%$ CI $0.675,0.745)$ and 0.709 (95\% CI 0.687 , 0.732 ), respectively, despite different overall insulin use between the two time periods (20.8\% and $38.2 \%$, respectively).

Data from a total of 1040 women in a separate clinic population (Liverpool Hospital Diabetes Centre, NSW Australia) were also used to test the model. This population was diagnosed at a mean of $23.0 \pm 6.9$ weeks gestation and had a mean age of 31.5 \pm 5.3 years, a mean OGTT fasting BGL of $5.2 \pm 0.9 \mathrm{mmol} / \mathrm{l}$, a mean $\mathrm{HbA}_{1 \mathrm{c}}$ of $5.4 \pm 0.56 \%(36 \pm 6.1 \mathrm{mmol} / \mathrm{mol})$ and a prepregnancy BMI of $27.9 \pm 6.9 \mathrm{~kg} / \mathrm{m}^{2}$. The ethnic background of this population was: East and South-East Asian, 22.4\%; Middle Eastern, 23.8\%; European, 23.6\%; South Asian, 20.8\%; and African subcontinent or Pacific Islander, 7.2\%. A total of 23 women $(2.2 \%)$ had missing data. All of these data differed significantly from those of the patient population in the present study ( $p<0.01$ for all). The model was found to be equally predictive of therapy type, as shown in Table 2 , despite the different model of care, significantly different ethnic composition $(p<0.0001)$ and different percentage insulin use $(47.2 \%$ vs $32.1 \%, p<0.0001)$. The AUC for the 'test site' ROC was very similar, at 0.707 (95\% CI 0.676, 0.739).

The number of predictors present was also found to be associated with the likelihood of several adverse perinatal outcomes (Table 3 ). Compared with women with $0-1$ predictors (low risk), women with 2-5 predictors (intermediate risk) or 6-7 predictors (high risk) were significantly more likely to have Caesarean delivery, an LGA infant and an abnormal postpartum OGTT, and early delivery was significantly higher for women with 6-7 vs 0-1 predictors. However, there were no differences in neonatal outcomes, including neonatal hypoglycaemia and shoulder dystocia. Similarly, as shown in Table 4, the number of predictors present also resulted in significantly different rates of Caesarean delivery and LGA infants (6-7 vs $0-1$ predictors), but not of early delivery in the 'test site' data. No data were available for neonatal outcomes or postpartum OGTT results at the 'test site'.

\section{Discussion}

The current study has successfully developed and validated a model that predicts future therapy type at diagnosis for women with GDM, using a number of readily available dichotomised variables found to be independent predictors of MNT+I, including both clinical and biochemical characteristics. The greater the number of predictors present $(0-7)$, the increased likelihood there was of requiring $\mathrm{MNT}+\mathrm{I}$. Conversely, the fewer predictors present, the greater the likelihood there was of women remaining on MNT alone. In addition, a higher number of predictors also increased the likelihood of several adverse perinatal outcomes, including Caesarean delivery and an LGA infant.

Consistent with the current study, a number of other studies have identified clinical and biochemical variables associated with a need for insulin therapy in GDM [3-12]. The majority of these identified independent predictors of therapy type using multivariable logistic regression analysis [3-9, 12]. Many identified the same predictors of insulin that were found in the present study, such as elevated pre-pregnancy BMI [4, $5,8,9]$, elevated OGTT fasting glucose [3, 4, 6, 8, 9], elevated 
Table 3 Number of predictors and corresponding occurrence of outcomes Bankstown-Lidcombe Hospital Diabetes Centre, NSW Australia

\begin{tabular}{|c|c|c|c|}
\hline \multirow[t]{2}{*}{ Outcome } & \multicolumn{3}{|c|}{ Number of predictors present } \\
\hline & $\begin{array}{l}0-1 \\
(n=805[24.3 \%])\end{array}$ & $\begin{array}{l}2-5 \\
(n=2399[72.3 \%])\end{array}$ & $\begin{array}{l}6-7 \\
(n=113[3.4 \%])\end{array}$ \\
\hline Early delivery ( $<37$ weeks) & $43 / 805(5.3)$ & $123 / 2399(5.1)$ & $14 / 113(12.4)^{*}$ \\
\hline Caesarean delivery & $160 / 803(19.9)$ & $640 / 2394(26.7) * * * *$ & $48 / 113(42.5)^{* * * *}$ \\
\hline Hypoglycaemia & $31 / 705(4.4)$ & $98 / 2152(4.5)$ & 9/102 (8.8) \\
\hline Shoulder dystocia & 4/705 (0.6) & $15 / 2152(0.7)$ & $1 / 102(1.0)$ \\
\hline Jaundice & $80 / 705(11.3)$ & $190 / 2152(8.8)$ & 9/102 (8.8) \\
\hline Hypoglycaemia and jaundice & $10 / 705(1.4)$ & $34 / 2152(1.6)$ & $0 / 102(0)$ \\
\hline SGA (<10th percentile) & 63/804 (7.8) & $217 / 2396(9.0)$ & $5 / 112(4.5)$ \\
\hline LGA (>90th percentile) & $79 / 803(9.8)$ & $358 / 2396(14.9)^{* * *}$ & $33 / 112(29.5) * * * *$ \\
\hline Abnormal OGTT post-partum ${ }^{a}$ & $86 / 471(18.3)$ & $365 / 1297(28.1) * * * *$ & $25 / 49(51.0)^{* * * *}$ \\
\hline \multicolumn{4}{|c|}{$\begin{array}{l}\text { Data are } n / \mathrm{XX}(\%) \text {, where the denominator is the number of patients with data available for each outcome } \\
\text { a At } 6-8 \text { weeks }\end{array}$} \\
\hline \multicolumn{4}{|c|}{$0-1$ predictor vs $2-5$ predictors: $* * * p<0.001, * * * * p<0.0001$} \\
\hline \multicolumn{4}{|c|}{$0-1$ predictor vs $6-7$ predictors: $* p<0.05, * * * * p<0.0001$} \\
\hline
\end{tabular}

$\mathrm{HbA}_{1 \mathrm{c}}$ at GDM diagnosis [3, 5, 9], a history of GDM in a previous pregnancy [9], a family history of type 2 diabetes [3, 5] and an earlier diagnosis of GDM [3, 7, 8]. Similar to in the present study, González-Quintero et al [8] and McFarland et al [7] found that a fasting glucose OGTT value of $>5.3 \mathrm{mmol} / 1$ was an independent predictor of the need for insulin therapy.

Consistent with this study, several other studies have also found elevated OGTT values (other than fasting BGL) to be independent predictors of a need for insulin therapy. Previous studies reported an elevated $1 \mathrm{~h}$ BGL on the OGTT to be an independent predictor [3, 4, 7, 9], whilst Wong et al (2011) found that an elevated $2 \mathrm{~h}$ OGTT value was an independent predictor for a requirement for insulin therapy [4]. However the latter finding was not consistent with the current study. In the present study, an elevated $2 \mathrm{~h}$ value was only predictive of insulin therapy in the univariate analysis, but not in the multivariable analysis. Hence, this variable was excluded from the final model.

Table 4 Number of predictors and corresponding occurrence of outcomes Liverpool Hospital Diabetes Centre, NSW Australia $^{a}$
Age (>30 years) was identified as a predictor of insulin therapy in the current study. This contrasted with the findings of a large study $(n=2365)$ by González-Quintero et al [8]. These authors concluded that laboratory values (namely, fasting $\mathrm{BGL}$ and $\mathrm{HbA}_{1 \mathrm{c}}$ ) were stronger predictors of insulin requirement compared with any patient characteristic.

Despite a number of studies identifying individual predictors of therapy type in GDM, only three studies have attempted to use such predictors to develop a model to estimate the likelihood of insulin therapy in an individual patient $[3,5,11]$. Pertot et al attempted to identify patients with GDM who would need antenatal insulin treatment using a riskprediction tool based on maternal clinical and biochemical characteristics at diagnosis [3]. However, these authors could only explain $9 \%$ of the variance in requiring insulin with the clinical variables included in their analysis, compared with $20.3 \%$ in the current study. They therefore concluded that a risk scoring system was a poor predictor of insulin therapy. In

\begin{tabular}{llll}
\hline Outcome & \multicolumn{2}{l}{ Number of predictors present } \\
\cline { 2 - 4 } & $\begin{array}{lll}0-1 \\
(n=219[21.1 \%])\end{array}$ & $\begin{array}{l}2-5 \\
(n=749[72.0 \%])\end{array}$ & $\begin{array}{l}6-7 \\
(n=72[6.9 \%])\end{array}$ \\
\hline Early delivery $(<37$ weeks) & $1 / 219(0.5)$ & $9 / 749(1.2)$ & $1 / 72(1.4)$ \\
Caesarean delivery & $37 / 219(16.9)$ & $187 / 749(25.0)^{*}$ & $27 / 72(37.5)^{* * *}$ \\
SGA $(<10$ th percentile) & $24 / 216(11.1)$ & $87 / 725(12.0)$ & $2 / 70(2.9)$ \\
LGA $(>90$ th percentile) & $20 / 216(9.3)$ & $110 / 725(15.2)^{*}$ & $19 / 70(27.1)^{* * *}$ \\
\hline
\end{tabular}

Data are $n / \mathrm{XX}(\%)$, where the denominator is the number of patients with data available for each outcome

${ }^{a}$ No neonatal outcomes or follow up OGTT data were available for this study site

$0-1$ predictor vs $2-5$ predictors: $* p<0.05$

$0-1$ predictor vs $6-7$ predictors: $* * * p<0.001$ 
contrast, Sapienza et al estimated the probability of insulin therapy based on the pre-pregnancy BMI, family history of diabetes, the number of abnormal $100 \mathrm{~g} 3 \mathrm{~h}$ OGTT values and $\mathrm{HbA}_{1 \mathrm{c}}$ at diagnosis [5]. However, these findings were based on a small sample (294 patients) and did not produce a simple clinically useful prediction tool. Mendez-Figueroa et al also attempted to predict the need for medical therapy in patients diagnosed with mild GDM (fasting BGL $<5.27 \mathrm{mmol} / \mathrm{l}$ in a $3 \mathrm{~h} 100 \mathrm{~g}$ OGTT) [11]. They constructed a predictive model with variables of significance including the fasting BGL on the OGTT, pre-pregnancy BMI and BMI at 26-30 weeks. When these variables were combined, a predictive model with a sensitivity of $76 \%$, a specificity of $52 \%$, a PPV of $48 \%$ and an NPV of $78 \%$ was produced. The PPV for requiring medical therapy in the study by Mendez-Figueroa et al was much lower than that in the present study (48\% vs $86.6 \%$ respectively), despite their slightly higher prevalence of patients requiring medical therapy (39\% vs $32.1 \%$ ) [11]. However, the differences between PPVs could be attributable to differences in the specificities of the two studies. The very high specificity found in the present study (99.4\%) will have led to a high PPV. Mendez-Figueroa et al concluded that antenatal factors, either alone or in combination, did not enable the possible need for medical therapy to be predicted in women with mild GDM.

The current study is novel. In contrast to previous studies, variables identified as being associated with insulin therapy in women with GDM were used to develop a model, which was then validated in a separate clinical population. The strength of this prediction model is indicated by its high specificity and PPV. The model performed well in predicting the requirement for $\mathrm{MNT}+\mathrm{I}$ in women with a high prediction score (6-7), and for predicting MNT only in women with a low score $(0-1)$. The OR further demonstrated the strength of the association in women who had 0-1 predictors and remained on MNT alone, and in women who had 6-7 predictors and commenced MNT+I.

The key aim of the model was to identify women who were unlikely to require insulin. This was accurately predicted by a low score, and characterised by the model's high specificity and PPV. The model's low sensitivity and NPV are not surprising because many women with a higher predictor score remained on MNT only.

The model developed in this study uses easily accessible clinical and biochemical variables that can be quickly tallied to determine individual risk, and therefore the level of monitoring and medical management required. Future research efforts will pilot this model to identify women with $0-1$ predictors and, after initial education and monitoring, refer them to a low-risk clinic with a predefined set of criteria for referral back to a higher risk clinic, with the expectation that up to $15 \%$ may need insulin therapy (see Table 2). Individuals with 2-7 predictors will be monitored more closely - because of the high likelihood that they will require insulin therapy and their greater risk of adverse pregnancy outcomes.
Whilst metformin was not used in this patient cohort, it would be appropriate to assess this model in a GDM cohort managed with metformin, which is used in some services as initial treatment in preference to insulin. The authors expect that the model would yield similar findings, but this needs to be tested.

This study has several limitations. First, as the study cohort included women of mixed ethnic background at a significant socioeconomic disadvantage and with a high GDM risk, the findings may not be generalisable to all women with GDM. Although the model was found to be similarly predictive in a different clinical population, the 'test site' cohort had similar ethnicities, although these were represented in significantly different proportions. It is yet to be determined whether the model would be similarly predictive in a clinical population with an entirely different ethnic mix. A second limitation was the retrospective observational study design which was reliant on routinely collected clinical data. Hence, several factors could have affected which final therapy type was chosen, including differences in clinical practice during the study period. It is also unknown whether this model would work with diagnostic criteria and/or treatment targets different from the ones used in the current study.

This study has a number of strengths. First, it had the largest dataset of any published analysis that has attempted to predict therapy type in women with GDM. Second, the GDM clinic was supervised by the same endocrinologist (JRF) for the entire study period. Further, the same diagnostic criteria and treatment targets were used throughout. In addition, standardised protocols were followed throughout the study period to minimise differences in clinical practice. Other strengths include validation in another clinical setting and the strength of the observed ORs, specificity and PPVs.

\section{Conclusion}

For the first time, an easy to use clinical prediction model has been developed which can be used within a busy outpatient clinical setting to assess women and assist with triaging into higher and lower risk clinics. Using this model, almost a quarter $(24.3 \%)$ of women with GDM in our clinic were assessed as being at low risk of MNT+I $(0-1$ predictors present) and considered for management in a low-risk setting. This is a significant finding in view of the rapidly rising GDM rates and the resultant constraints on available staff and financial resources. Triaging such a significant proportion of the GDM clinic population into a low-risk model of care could allow additional clinic time for more intensive monitoring and medical management of women deemed to be at higher risk of adverse outcomes. Although the model was found to have moderate predictive power in two clinical cohorts, further research is needed to determine whether it is equally predictive 
in other clinic populations. Future piloting of the model could be conducted within a new model of care, in which it could be evaluated according to pregnancy outcomes and resource utilisation.

Acknowledgements We wish to thank the following diabetes educators who have collected data and maintained the database over the 23 years of data collection: N. Edghill, C. Finneran, C. Fung, M. Griffiths, G. Holters, M. Horne, J. Mackenzie, R. Myszka, A. O’Neill, A. Oni, J. Payne, M. Stephens, C. van Geloven, J. Warrender and A. Watt, all from the Diabetes Centre, Bankstown-Lidcombe Hospital, Australia.

We would also like to thank J. Gardosi, West Midlands Perinatal Institute, Birmingham, UK, for use of the customised percentile chart calculator. We also thank G. S. Gabriel, Ingham Institute, Liverpool Hospital, Australia, for his assistance with the intricacies of the SPSS program.

The findings in this paper were presented at the ADIPS Annual Scientific Meeting Adelaide, South Australia, 28-30 August 2015, and published in the conference proceedings: abstract 21 [19].

Funding This research received no specific grant from any funding agency in the public, commercial or not-for-profit sectors.

Duality of interest statement The authors declare that there is no duality of interest associated with this manuscript.

Contribution statement RAB was responsible for study conception. CEC, CES, GRP, JRF, RAB and TW were responsible for study design; RAB performed background research; CEC, GPR, JRF and VWW provided administrative and technical support; JRF provided material support; CEC, CES, JRF and LM-W were involved in supervising the study; JRF and VWW were responsible for data acquisition; BBJ and TW provided statistical expertise; BBJ, JRF and TW analysed the data; BBJ, CEC, CES, GPR JRF, LM-W, RAB and TW interpreted the data; RAB and JRF wrote the manuscript; RAB edited the manuscript; and BBJ, CEC, CES, GPR, JRF, LM-W, TW and VWW critically revised the manuscript. All authors approved the final version of this manuscript. JRF is the guarantor of this work.

\section{References}

1. Crowther CA, Hiller JE, Moss JR et al (2005) Effect of treatment of gestational diabetes mellitus on pregnancy outcomes. N Engl J Med 352:2477-2486

2. Metzger BE, Buchanan TA, Coustan DR et al (2007) Summary and recommendations of the Fifth International Workshop-Conference on Gestational Diabetes Mellitus. Diabetes Care 30:S251-S260
3. Pertot T, Molyneaux L, Kris T et al (2011) Can common clinical parameters be used to identify patients who will need insulin treatment in gestational diabetes mellitus. Diabetes Care 34:2214-2216

4. Wong V, Jalaludin B (2011) Gestational diabetes mellitus: who requires insulin therapy? Aust N Z J Obstet Gynaecol 51:432-436

5. Sapienza AD, Fransisco RPV, Trindade TC, Zugaib M (2010) Factors predicting the need for insulin therapy in patients with gestational diabetes mellitus. Diabetes Res Clin Pract 88:81-86

6. Akinci B, Celtik A, Yener S, Yesil S (2008) Is fasting glucose during oral glucose tolerance test an indicator of the insulin need in gestational diabetes? Diabetes Res Clin Pract 82:219-225

7. McFarland MB, Langer O, Conway DL, Berkus MD (1999) Dietary therapy for gestational diabetes: how long is long enough? Obstet Gynecol 93:978-982

8. González-Quintero VH, Istwan NB, Rhea DJ et al (2008) Antenatal factors predicting subsequent need for insulin treatment in women with gestational diabetes. J Women's Health 17:1183-1187

9. Mitra S, Nayak PK, Sahoo J et al (2014) Predictors of antenatal insulin requirement in gestational diabetes. Gynecol Endocrinol 30:565-568

10. Tan YY, Liauw PCY, Yeo GSH (2008) Using glucose tolerance test results to predict insulin requirement in women with gestational diabetes. Aust N Z J Obstet Gynaecol 3:262-266

11. Mendez-Figueroa H, Daley J, Lopes VV, Coustan D (2014) Predicting the need for medical therapy in patients with mild gestational diabetes. Am J Perinatol 31:105-112

12. Bakiner O, Bozkirli Ozsahin K, Sariturk C, Ertorer (2013) Risk factors that can predict antenatal insulin need in gestational diabetes. J Clin Med Res 5:381-388

13. Australian Bureau of Statistics (ABS) (2011) Census data. www. censusdata.abs.gov.au/census services/getproduct/census/2011 /quickstat/LGA10350?opendocument\&navpos=220. Accessed 4 Sept 2015

14. Martin FIR for the Ad Hoc Working Party (1991) The diagnosis of gestational diabetes. Med J Aust 155:112

15. Hoffman L, Nolan C, Wilson JD, Oats JJN, Simmons D (1998) Gestational diabetes mellitus management guidelines. The Australasian Diabetes In Pregnancy Society. MJA 169:93-97

16. Institute of Medicine (USA) (1990) Subcommittee on nutritional status and weight gain during pregnancy and subcommittee on dietary intake and nutrient supplements during pregnancy, nutrition during pregnancy. National Academic Press, Washington DC

17. Institute of Medicine. Weight Gain During Pregnancy: Reexamining The Guidelines. Report Brief May 2009. National Academies Press, Washington DC

18. Gardosi J, Francis A (2014) Customised Weight Centile Calculator - GROW Gestation Network, available from www.gestation. net/index.htm bulk centile calculator v6.7.3_13. Accessed 1 Feb 2014

19. Barnes RA, Wong T, Ross GP et al (2015) A model for the prediction of therapy type in women with gestational diabetes mellitus. Abstract 21, Proceedings of the Australasian Diabetes In Pregnancy Society Annual Scientific Meeting, August 2015 Adelaide, South Australia 